uPAR, elevated levels may reflect increased activation of immune system which observed in the autoimmune diseases.

Objectives: The aim of this study was to assess the effects of anti-TNF therapy on uPAR production in rheumatoid arthritis (RA) and ankylosing spondylitis (AS). We also wished to correlate suPAR levels with various autoimmune-inflammatory biomarkers.

Methods: Altogether 33 arthritis patients including 22 RA patients treated with either etanercept (ETN) or certolizumab pegol (CZP) and 11 AS patients treated with ETN were included in a 12- month follow-up study. Circulating suPAR levels were assessed by suPARnostic Quick Test Reader. In addition,disease activity (DAS28 or BASDAI), CRP, IgM rheumatoid factor, anti-CCP were also assessed. Assessment were performed at baseline, as well as 6 and 12 months after treatment initation.

Results: Anti-TNF treatment was highly effective in both disease, as the mean DAS28 decreased in RA, mean BASDAl decresead in AS. There were no significant change in the suPAR levels after 12 months of anti-TNF therapy, although resulted non-significant decrease $(p<0,18)$ in RA patients with critical suPAR levels ( $>9 \mathrm{ng} / \mathrm{ml}$ ). Baseline suPAR levels positively correlated with anti-CCP $(p<0,001)$ and rheumatoid factor $(p=0,024)$ in RA patients. Circulating suPAR levels did not correlate with DAS28, BASDAI or CRP.

Conclusions: In a mixed cohort of RA and AS patients, anti-TNF therapy did not affect the suPAR levels after 12 months. suPAR levels correlated with rheumatoid factor and anti-CCP. Based on these results suPAR may be a marker of autoimmunity rather than that of disease activity.

Disclosure of Interest: None declared

DOI: 10.1136/annrheumdis-2017-eular.3428

\section{THU0695 THE RA FLARE QUESTIONNAIRE (RA-FQ) IS RESPONSIVE TO CHANGE IN RA SYMPTOMS AND IMPACTS IN CLINICAL AND OBSERVATIONAL TRIALS}

S.J. Bartlett $^{1,2}$, V.P. Bykerk ${ }^{3}$, B. Fautrel ${ }^{4}$, F. Guillemin ${ }^{5}$, A. den Broeder ${ }^{6}$, R. Alten ${ }^{7}$, R. Christensen ${ }^{8}$, E. Choy ${ }^{9}$, D. Furst ${ }^{10}$, S. Hewlett ${ }^{11}$, A. Leong ${ }^{12}$ L. March ${ }^{13}$, T. Woodworth ${ }^{10}$, B.I.O. Clifton ${ }^{1}$ on behalf of CATCH, STPR, \& DRESS Investigators. ${ }^{1}$ Johns Hopkins, Baltimore, United States; ${ }^{2}$ McGill University, Montreal, Canada; ${ }^{3}$ HSS, New York, United States; ${ }^{4}$ Pierre \& Marie Curie Universite, Paris; ${ }^{5}$ Universite de Lorraine, Nancy, France; ${ }^{6}$ Maartens Kliniek, Nijmegen, Netherlands; ${ }^{7}$ Schlosspark Klinik, Berlin, Germany; ${ }^{8}$ Parker Institute, Copenhagen, Denmark: ${ }^{9}$ Cardiff University, Cardiff, United Kingdom: ${ }^{10}$ UCLA, Los Angeles, United States; ${ }^{11}$ University of the West of England, Bristol, United Kingdom; ${ }^{12}$ Healthy Motivation, Santa Barbara, United States;

${ }^{13}$ University of Sydney, Sydney, Australia

Background: The RA-FQ is a new tool to measure RA flares. We have previously provided evidence of construct validity and reliability. 1

Objectives: We evaluated sensitivity to change of the RA-FQ in clinical trials and observational studies of RA patients with low disease activity.

Methods: RA patients in observational studies [CATCH-Canada ( $n=896)$ and STPR-France $(n=138)$ ], and an RCT (DRESS-Netherlands; $n=178$ ) completed 5 items asking whether they were in flare (yes/no), and if so, the severity and duration. We selected patients who said they were not in flare and had a DAS28 $<3.2$ at thefirst visit (V1). Flare at the next study visit (V2) was defined three ways: 1) patient report (yes/no); 2) patient report-stringent (Boolean: patient report yes AND severity $\geq 4 / 10$ AND duration $>7$ days [to increase this reflected increased inflammatory disease activity]; and c) DAS definition often used in studies (DAS28 $<3.2$ at V2 required increase of 1.2 ; DAS $\geq 3.2$ at V2 required increase of 0.6 ). We compared RA-FQ scores with other PROs and disease activity indicators between flaring and non-flaring patients at V2. Effect size was estimatedusing Cohen's SMD. We hypothesized that at V2, RA-FQ scores would be similar in patients not flaring at both visits, and would be significantly higher in those flaring at V2.

Results: The mean difference in RA-FQ scores at V2 ranged from $7.3(95 \% \mathrm{Cl}$ $1.4,13.2)$ in STPR (DAS def) to $19.6(95 \% \mathrm{Cl} 16.7,22.6)$ in $\mathrm{CATCH}$ (patient report-stringent). The standardized mean difference effect sizes (SMDES) ranged from $0.82-1.95$, and were largest for patient report-stringent in 2 studies. SMDE

\begin{tabular}{|c|c|c|c|c|c|c|}
\hline & \multicolumn{2}{|c|}{$\begin{array}{c}\text { Flare } \\
\text { (Second Visit) }\end{array}$} & \multicolumn{2}{|c|}{$\begin{array}{c}\text { No Flare } \\
\text { (Second Visit) }\end{array}$} & \multirow{2}{*}{$\begin{array}{c}\text { Mean difference } \\
\text { RA-FQ Score } \\
(95 \% \mathrm{Cl})\end{array}$} & \multirow{2}{*}{$\begin{array}{l}\text { Effect } \\
\text { Size }\end{array}$} \\
\hline & V1 & V2 & V1 & V2 & & \\
\hline \multicolumn{7}{|l|}{$\mathrm{CATCH}^{1}$ (Canada) } \\
\hline Patient Report & 13.4 & 22.5 & 9.8 & 8.5 & $14.0(11.4,16.5)$ & 1.39 \\
\hline Patient Report - Stringent ${ }^{\star}$ & 16.6 & 29.0 & 9.9 & 9.4 & $19.6(16.7,22.6)$ & 1.95 \\
\hline DAS-defined flare $\neq$ & 11.8 & 26.8 & 10.0 & 9.3 & $17.5(13.6 .21 .5)$ & 1.73 \\
\hline \multicolumn{7}{|l|}{ DRESS$^{2}$ (Netherlands) } \\
\hline Patient Report & 17.4 & 22.4 & 11.6 & 12.2 & $10.2(5.6,14.8)$ & 1.10 \\
\hline Patient Report - Stringent ${ }^{*}$ & 16.7 & 31.3 & 12.2 & 12.9 & $18.3(8.9,27.8)$ & 1.10 \\
\hline DAS-defined flare $*$ & 187 & 24.6 & 11.6 & 12.2 & $12.4(7.7,17,2)$ & 1.37 \\
\hline \multicolumn{7}{|l|}{ STPR $^{3}$ (France) } \\
\hline Patient Report & 17.5 & 22.7 & 13.0 & 13.0 & $9.6(3.6,15.6)$ & 1.10 \\
\hline Patient Report - Stringent* & 17.1 & 25.3 & 13.3 & 13.7 & $11.5(23,20.8)$ & 1.30 \\
\hline DAS-defined flare $\neq$ & 16.5 & 20.5 & 13.9 & 13.2 & $7.3(1.4,13.2)$ & 0.82 \\
\hline \multicolumn{7}{|c|}{$\begin{array}{l}\text { Canadian Early Arthritis Cohort, } 2 \text { Dose Reduction Strategy of Subcutaneous TNF Inhibitors in Rheumatoid Arthritis; } \\
3 \text { Strategy of Treatment in Patients with Rheumatoid Arthritis } \\
\text { 'Stringent }=\text { Boolean: patient report flare yes AND severity } \geq 4 / 10 \text { AND duration }>7 \text { days. } \\
\text { "DAS28 <3.2 at V2 required increase of 1.2; DAS } \geq 3.2 \text { at V2 required increase of } 0.6\end{array}$} \\
\hline
\end{tabular}

were strong (range 0.84-2.42) for patient global, MD global, HAQ, DAS28, and other clinical indicators except ESR.

The mean difference in RA-FQ scores at V2 ranged from $7.3(95 \% \mathrm{Cl} 1.4,13.2)$ in STPR (DAS def) to $19.6(95 \% \mathrm{CI} 16.7,22.6)$ in $\mathrm{CATCH}$ (patient report-stringent). The standardized mean difference effect sizes (SMDES) ranged from 0.82-1.95, and were largest for patient report-stringent in 2 studies. SMDE were strong (range 0.84-2.42) for patient global, MD global, HAQ, DAS28, and other clinical indicators except ESR.

Conclusions: Data from clinical and observational studies support the responsiveness of the RA-FQ in detecting change over time. The robust psychometric properties of the RA-FQ suggest it reliably detects clinically relevant worsening of RA symptoms consistent with increased disease activity and support its use in research and clinical care.

References:

[1] Bykerk V et al. RMD Open 2016;2(1):e000225.

Disclosure of Interest: None declared

DOI: 10.1136/annrheumdis-2017-eular.6921

\section{THU0696 ANTI-CARBAMYLATED PROTEINS ANTIBODIES IN SLE PATIENTS WITH JOINT INVOLVEMENT: A POSSIBLE NEW BIOMARKER FOR EROSIVE DAMAGE}

F. Ceccarelli $^{1}$, C. Perricone ${ }^{2}$, L. Massaro ${ }^{1}$, T. Colasanti ${ }^{1}$, E. Cipriano ${ }^{1}$, M. Pendolino ${ }^{1}$, F. Natalucci ${ }^{1}$, G. Capalbo ${ }^{1}$, R. Mancini ${ }^{1}$, F.R. Spinelli ${ }^{1}$, C. Alessandri ${ }^{1}$, G. Valesini ${ }^{1}$, F. Conti ${ }^{1} .{ }^{1}$ Lupus Clinic, Reumatologia, Dipartimento di Medicina Interna e Specialità Mediche, Sapienza Università di Roma, Roma; ${ }^{2}$ Lupus Clinic, Reumatologia, Dipartimento di Medicina Interna e Specialità Mediche, Sapienza Università di Roma, Rome, Italy

Background: The traditional concept of non-erosive arthritis in Systemic Lupus Erythematosus (SLE) patients changed during the last years, thanks to the use of more sensitive imaging techniques, such as ultrasonography (US). The predictive role of Rheumatoid Arthritis (RA)-specific autoantibodies for bone loss in SLE patients has been investigated. In particular, anti-citrullinated peptide antibodies (ACPA) have been identified in about $50 \%$ of SLE patients with $\mathrm{x}$-Ray detected erosive arthritis. More recently, anti-carbamylated proteins (anti-CarP) antibodies have been demonstrated in seronegative RA patients, with a prevalence of about $16 \%$ and a significant association with erosive damage.

Objectives: In the present cross-sectional study, we aimed at assessing the association between anti-CarP antibodies and erosive damage in a large cohort of SLE patients with joint involvement.

Methods: For this purpose, we evaluated 152 SLE patients (1997 ACR criteria; $M / F 11 / 141$, mean $\pm S D$ age $46.4 \pm 11.3$ years, mean $\pm S D$ disease duration $144.9 \pm 110.5$ months) with joint involvement (arthralgia/arthritis). The clinical and laboratory data were collected in a standardized computerized electronically filled form. All patients underwent blood draws to detect Rheumatoid Factor (RF) and ACPA, by using commercial ELISA kits according to the manufacturer's instructions, and anti-CarP antibodies by home-made ELISA (results were expressed in arbitrary units $(\mathrm{AU}) / \mathrm{ml}$ and values above $340 \mathrm{IU} / \mathrm{ml}$ were considered positive). US was performed to assess the bone surfaces of metacarpophalangeal and proximal interphalangeal. At each joint, according with OMERACT definition, the presence of erosions was registered with a dichotomous value $(0 / 1)$, allowing the possibility to obtain a total score, ranging from 0 to 20 .

Results: The prevalence of anti-CarP antibodies was $28.3 \%$, similar to RF $(27.6 \%)$ and significantly higher to ACPA $(11.2 \%, p=0.003)$. The mean \pm SD titer of anti-CarP antibodies was $890.5 \pm 794.9 \mathrm{IU} / \mathrm{ml}$. Thirty-nine patients $(25.6 \%)$ showed erosive arthritis: all the patients referred the occurrence of at least one episode of clinical synovitis. Erosive arthritis was significantly associated with anti-CarP antibodies $(p=0.004)$ and ACPA $(p=0.0008)$. Moreover, a significant correlation between anti-CarP antibody titer and US-erosive score was observed $(r=0.2$, $\mathrm{p}=0.01$ ). Of note, anti-CarP antibodies were identified in $24.5 \%$ of double negative (ACPA-/RF-) patients, with erosive damage in $25 \%$ of them. Interestingly, antiCarP antibodies resulted significantly associated with the presence of anti-dsDNA $(\mathrm{P}=0.01)$.

Conclusions: In the present study, for the first time, we identified a significant association between anti-CarP antibodies and erosive damage in SLE-related arthritis, in terms of frequency and severity. We found these antibodies in about $25 \%$ of $\mathrm{ACPA}^{-} / \mathrm{RF}^{-}$SLE patients, a prevalence higher than that described in seronegative RA patients. Taken together, our results suggest that anti-CarP antibodies could be considered as a candidate biomarker of severity in SLE patients with joint involvement.

Disclosure of Interest: None declared

DOI: 10.1136/annrheumdis-2017-eular.5307 